\title{
A rare case of bilateral basal cell adenomas in the parotid glands
}

\author{
K. Anji Reddy $\cdot$ A. Trimurthy Rao $\cdot$ R. Krishna $\cdot$ Y. Manjula $\cdot$ M. Sambasiva Rao $\cdot$ K. Srinivasulu
}

Received: 20 January 2007 / Accepted: 24 January 2008

\begin{abstract}
We report a very rare case of bilateral parotid tumors in a 55-year-old female patient who presented with left parotid mass of $7 \times 7 \mathrm{~cm}$ size since 8 years and right parotid mass of $2.5 \times 1.5 \mathrm{~cm}$ size since 2 years duration. Based on clinical features a provisional diagnosis of bilateral pleomorphic adenomas was made. Results of fine needle aspiration cytology of both masses were inconclusive. Bilateral superficial conservative parotidectomy with facial nerve preservation revealed bilateral encapsulated and lobulated tumors which on histopathological examination revealed bilateral basal cell adenomas in both parotid glands.
\end{abstract}

Keywords Bilateral parotid tumors - Basal cell adenoma - Parotid tumors

K. A. Reddy · A. T. Rao - R. Krishna - Y. Manjula · M. S. Rao ·

K. Srinivasulu

Department of Surgery and Pathology,

NRI Medical College and Hospital,

Chinakakani, Guntur,

India

Anji Reddy K. ( $₫)$

e-mail: reddykanji@rediffmail.com

\section{Introduction}

The bilateral occurrence of parotid tumors is rare and accounting for $1-3 \%$ of all parotid tumors. Most bilateral parotid tumors are Warthin's tumors and pleomorphic adenomas [1]. The occurrence of bilateral basal cell adenomas in the parotid glands is extremely rare and only five cases have been reported in the literature so far [1,2]. We report this uncommon and rare case in an old female patient. Surgical resection is associate with an excellent prognosis in basal cell adenomas. But present of membranous component in histological sections requires periodical check up for the possibility of any recurrence with malignant transformation [3]. The purpose of this case report is to plan further line of treatment as per the histological sub types in addition to its rarity.

\section{Case report}

A 55-year-old female patient was admitted because of palpable mass in the left parotid region since 8 years and a palpable mass in the right parotid region since 2 years. Physical examination revealed a non-tender, firm, movable swelling, measuring $7 \times 7 \mathrm{~cm}$ on left side (Fig. 1a) and $2.5 \times 1.5 \mathrm{~cm}$ on right side (Fig. 1b) on either side the deep lobe was not palpable, no evidence of any discharge from the duct orifice and no evidence of facial nerve palsy or regional lymphadenopathy. The movements of the jaws were normal. Routine laboratory investigations were within normal limits. Fine needle aspiration cytology (FNAC) reports were inconclusive. Bilateral superficial conservative parotidectomy was done with facial nerve preservation. The tumor was well encapsulated on either side. Squash (crush) cytology report from both freshly excised masses was bilateral monomorphic adenomas. 
On gross examination both left and right parotid masses were encapsulated with smooth and lobulated surface (Fig. 2). Gross resection of both masses showed well formed capsule, uniformly grey white solid tumor with minute cystic and minimal vascular areas (Fig. 2. inset). No necrosis was seen.

Microscopically both the masses showed identical histological picture of well encapsulated basal cell adenomas and its appearance was reminiscent of that of a skin adnexal tumor (Fig. 3) the tumor cells are cuboidal to columnar at the periphery with nuclear palisading. The second type of cells were larger and they formed most of central portions of the tumor nests. Both the adenomas showed solid, tubular, trabecular, and membranous sub types. Most of these subtypes dispersed in a scanty fibrous stroma.

The most common histological pattern seen in both the tumors were trabecular (Fig. 3) and solid types (75\%) rest of the areas showed tubular, canalicular and membranous types. The membranous type constituted about $5 \%$ in both
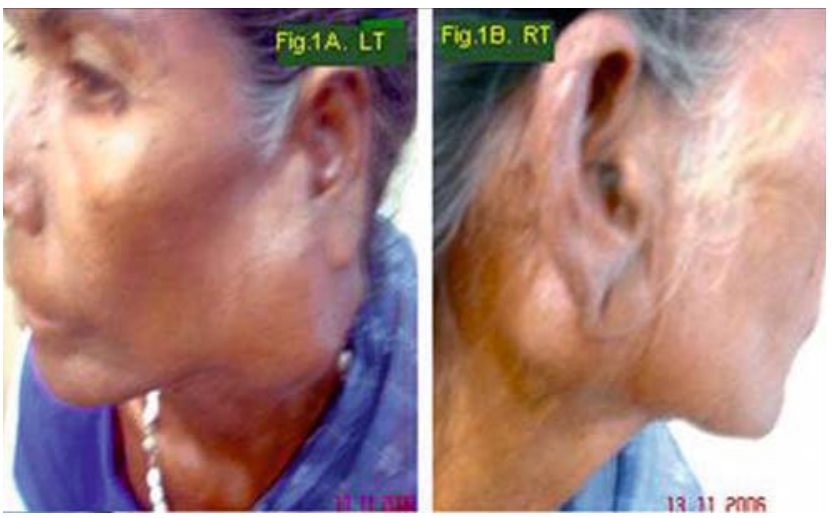

Fig. 1A,B Clinical photograph showing both left (A) and right (B) Parotid swellings.

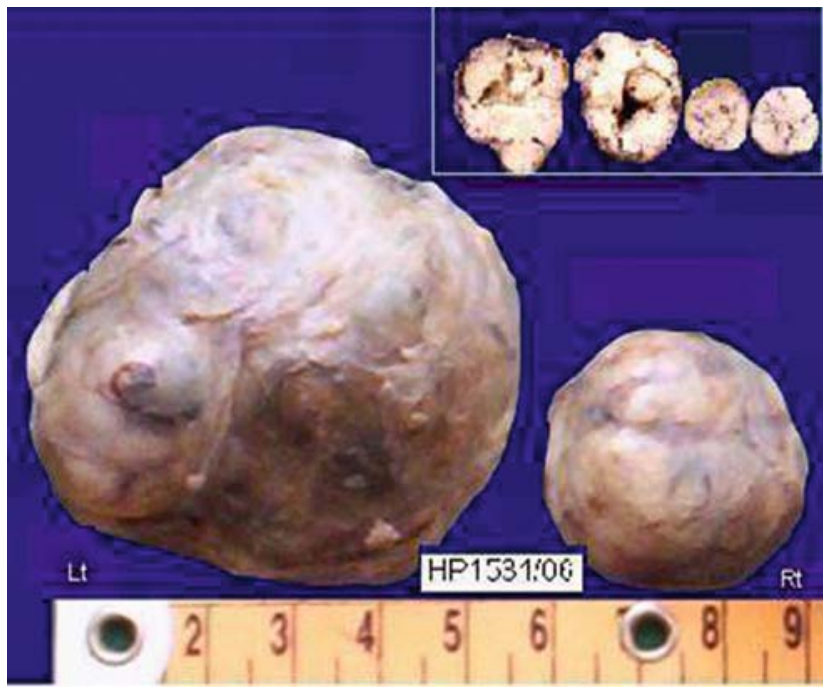

Fig. 2 Gross appearance of Basal Cell Adenomas of both left and right parotid glands. Inset: Cut section appearance of the same tumors

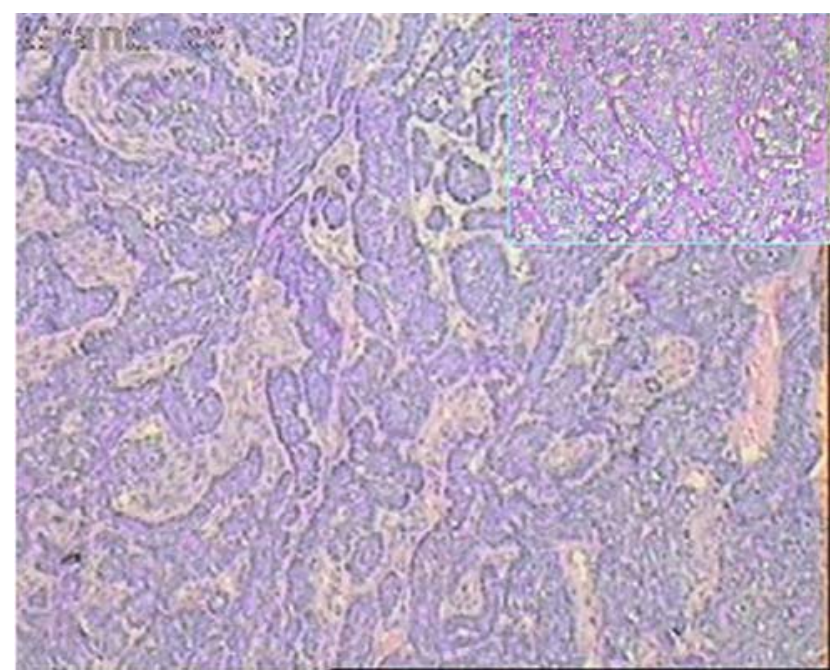

Fig. 3 Photographs of Basal Cell adenoma Parotid glands showing islands and trabeculae of basaloid cells. Palisading of tumor cells seen at the periphery $(\mathrm{H} \& \mathrm{E} \times 100)$.

Inset: Membranous type showing PAS +ve basement membrane material (PAS, $\times 100)$.

tumors and it was different from other sub types by the presence of thick eosinophilic PAS +ve hyaline basal lamina material around the tumor islands (Fig. 3).

Postoperative period was uneventful and patient was discharged with an advice to have periodical checkup, in view of note worthy presence of membranous sub type component in both the basal cell adenomas histologically and for the possibility of any recurrence with malignant transformation.

\section{Discussion}

By definition basal cell adenoma is a benign tumor composed of basaloid cells sharply delineated from the stroma by basement membranes. It usually exhibits a mixture of various histological patterns (sub types). The mesenchymal component or chondromyxoid stroma characteristic of pleomorphic adenoma will be absent [3]. These tumors composed of two types of cells. The first are small cells that are usually congregated at the periphery and commonly show nuclear palisading. The second type of the cells are larger, they are predominant and form most of the central portion of various histological sub types. Basal cell adenoma is an uncommon benign epithelial tumor of salivary gland accounting for $1-2 \%$ of all salivary gland epithelial tumors [4]. More than $80 \%$ arise in major salivary glands [5] and about $70 \%$ of these tumors occur in parotid glands [3] and arise mainly in adults and the average patient is being 57.7 years [5]. There is a $2: 1$ female predominance. An extremely unusual congenital form has been described which always must be distinguished from an embryoma. 
The occurrence of bilateral basal cell adenomas is extremely rare and most uncommon.

Approximately $70 \%$ of basal cell adenomas occur in the parotids [6]. The bilateral occurrence of parotid tumors is rare accounting for $1-3 \%$ of all parotid tumors. Most bilateral parotid tumors are Warthin's tumors and pleomorphic adenomas. The occurrence of bilateral basal cell adenomas of the parotid glands is very rare and only five cases have been reported so far. In contrast to these earlier five cases there are no coexisting dermal cylindromas or adenoid cystic carcinomatous areas in the present case. Review of literature did not reveal even a single case report of bilateral basal cell adenomas of parotid glands in India $[6,7]$.

The exact mechanism contributing to the development of bilateral parotid tumors remains unknown, it is speculated that there could be involvement of environmental and genetic factors since the histological features of both the parotid tumors are identical in most cases [2].

Ultrastructural studies suggest that basal cell adenoma, like most other salivary gland tumors, is derived from the intercalated portion of the duct with a minor participation of myoepithelial cells.

Recurrence is rare and almost nonexistent for solid, trabecular and tubular variants of basal cell adenoma except for the membranous sub type which is associated with a recurrence rate of $25 \%$, probably because of its multi focal nature and absence of capsule in $50 \%$ of them. Membranous basal cell adenoma also known as dermal analogue tumor, is a distinctive variant associated with cutaneous adenexal tumors such as dermal cylindroma, trichoepithelioma and eccrine spiradenoma [3]. Surprisingly unlike other subtypes membranous basal cell adenoma shows a $90 \%$ preponderance in males [3] and multi centric development in $50 \%$ of cases [3].
Basal cell adenoma may rarely undergo malignant transformation ( $4 \%$ ) where as the transformation rate is $28 \%$ in membranous sub type. The transformed carcinoma may be either basal cell adenocarcinoma or adenoid cystic carcinoma or non basaloid type of salivary duct carcinomas or adenocarcinoma [3].

In conclusion we have reported a very rare case of Bilateral Parotid Basal cell adenomas with various histological patterns including membranous subtype.

\section{References}

1. Suzuki S, Okamura H, Ohtani I (2000) Bilateral parotid gland basal cell adenomas. Case report. ORL Journal for Oto-rhinolaryngology and its Related Specialties, 62(5): 278-281

2. Santhoshi Kastuno, Ishii, Akishiro Otsuka, Susumu Exawa and Shin-Ichi Usami (2000) Bilateral basal cell adenomas in the parotid glands: Journal of Laryngology and Otology 114:83-85

3. Christopher DM (2003) Fletcher Diagnostic Histology of Tumors, 2nd ed. (Elsevier Sciences) Vol. I, pp. 245-247

4. Seifert G, Sobin LH (1991) Histological typing of salivary gland tumors. In WHO International Histological classification of Tumors, 2nd ed. (Springer Verlag, Berlin), pp. 20-21

5. Gnepp Dr, Brandwein MS, Henley JD (2000) Salivary and lacrimal glands. In Diagnostic Surgical Pathology of the Head and Neck, 1st ed. (Gnepp Dr. eds; W.B. Saunders, Philadelphia), pp. $325-430$

6. Shafkat A, Mohainmad L, Rouf A (2002) Clinicopathological study of primary salivary gland tumors in Kashmir: JK-practitioner 9(4): 231-233

7. Nitin M, Nagarkar, Sandeep B, Arjun D, Surinder K Singhal, Harsh Mohan (2004) Salivary gland tumors - our experiences: Indian journal of Otolaryngology and Head and Neck SurgeryVol. 56 No.1 